\title{
Bedeutung der Grundregulation für das Stress- geschehen unter besonderer Berücksichtigung von Neuraltherapie und Akupunktur*
}

\author{
Hartmut Heine \\ Professor für Anatomie, Histologie und Embryologie, ECM-Forscher, DE-Neuhausen
}

elastungsstörungen („funktionellsomatische Störungen“) wie Leistungsabfall und Erschöpfung (chronisches Müdigkeitssyndrom), viszerale Hyperalgesie (chronisches Reizdarmsyndrom oder Reizblase), Dysmenorrhoe (prämenstruelles Syndrom), Überempfindlichkeit gegen Umweltstoffe (multiple Chemikaliensensitivität), Panikattacken mit Angststörungen (posttraumatisches Syndrom PTSD) und soziale Vereitelungsprozesse (Mobbing) stellen eine zunehmende Crux für die ärztliche Praxis dar [3]. Es darf auch nicht übersehen werden, dass auch der Alterungsprozess Stesssymptomatik zeigt [12]. Charakteristisch ist, dass diese Störungen meist überlappend als „Cluster“ auftreten [1]. Besonders hoch ist die Koexistenz des Fibromyalgiemit dem Reizdarmsyndrom (bis zu $70 \%$ ) und dem PTSD (bis zu 56\%). Gut dokumentiert ist auch die Komorbidität mit Angststörungen, depressiven Verstimmungen oder Persönlichkeitsstörungen [3]. Derartige funktionellsomatische Störungen nehmen stark $\mathrm{zu}$, sie betrafen bereits vor 15 Jahren ca. $80 \%$ der Klientel einer Allgemeinpraxis [27].

Im Folgenden soll gezeigt werden, dass unter Beachtung des Systems der Grundregulation diese Problematik regulationstherapeutisch gut angehbar ist.

\footnotetext{
* Meinem lieben Freund Dr. med. Andreas Beck, dem Begründer der „Kollegialen Instanz für Komplementärmedizin (KIKOM)" an der Universität Bern und Mitbegründer des „European Council for Doctors for Plurality in Medicine (ECPM) (Brüssel)" zum 70. Geburtstag gewidmet. Ad multos annos!
}

Stress stellt immer eine unspezifische Reaktion des Körpers auf jegliche Belastung dar. Wird dieser Prozess chronisch, entwickeln sind daraus Belastungsstörungen, die die Crux jeder allgemeinärztlichen Praxis darstellen. Der dabei durch die Stressschiene Hypothalamus-Hypophyse-Nebenniere gestörte bio-psycho-soziale Funktionskreis hat ein neuroplastisches Korrelat im kontrollierten und unkontrollierten Stressverarbeitungsprozess. Dabei ist die Reaktionsfähigkeit der extrazellulären Matrix (ECM) von entscheidender Bedeutung, da sie alle Funktionen im bio-psychosozialen Funktionskreis beeinflusst. Dafür stehen als "Stresszügel“ zwei in der ECM ubiquitär vorhandene „Bremssubstanzen" zur Verfügung, die von immunkompetenten Zellen gebildet werden. Das nichtneuronale Azetylcholinsystem (NNAChS) und das Endocannabinoidsystem. Beide Systeme wirken regulierend auf den stressbedingt erhöhten Sympathikotonus und dadurch auch schmerzabbauend. Sie sind regulationstherapeutisch sehr gut über Akupunktur und Neuraltherapie beeinflussbar. Dabei hat sich gezeigt, dass Akupunktur u. a. den Parasympathikus und das NNAChS und Neuraltherapie vor allem das periphere Endocannabinoidsystem anregt und den Sympathikus hemmt.

Schlüsselwörter: Extrazelluläre Matrix, bio-psycho-sozialer Funktionskreis, Stressbewältigungsprozesse

\section{Regulation of the Extracellular Matrix: Significance for Stress Reactions particularly in Neuraltherapy and Acupuncture}

Stress is an unspecific reaction on each body load. If such a process becomes chronic, stressbased diseases develop which are hard to treat. The stress track hypothalamus-hypophysissuprarenal glands disturbes the bio-psycho-social circuit which feeds back with controlled and uncontrolled stress converting processes. This is coupled with the reactivity of the extracellular matrix (ECM) embedded in this circuit. Two "breaks" are installed in the ECM to keep stress reactions under control: The non-neuronal acetylcholin system (NNAChS) and the system of endocannabinoids. Both systems have a limiting effect on pathologically increased sympathetic effects raised by stress. Acupuncture stimulates the parasympathicus and NNAChS while neuraltherapy augments the endocannabinoid system slowing down sympathetic actions.

Keywords: Extracellular matrix, bio-psycho-social circuit, stress-relieving processes

\section{Stress und Grundregulation}

Prinzipiell lässt sich bei den Belastungsstörungen die von SELYE [29], dem Vater der Stressforschung (1907-1982), gegebene Definition von Stress als eine „unspezifische Reaktion des Körpers auf jegliche Belastung“ wiedererkennen. Die dabei aktivierte „Stressachse“ Hypothalamus-Hypophyse-Nebenniere (HHN-Achse) führt zu einer chronischen Belastung des Körpers vor allem durch Katecholamine, Cortisol und proinflammatorische Zytokine (Abb. 1).
Dadurch erhöhen sich die katabolen Prozesse und damit der Energiebedarf im Körper. Dies führt u. a. zu vermehrter Bildung von Sauerstoffradikalen (ROS, reactive oxygen species), wodurch der Entwicklung von Belastungsstörungen und den daraus resultierenden Zivilisationserkrankungen Vorschub geleistet wird. Belastungsstörungen bzw. Stress jeder Art sind daher immer auch mit Schmerzphänomenen verbunden [10, 11].

Wenn auch die Stressforschung immer genauere Hinweise auf ein $\mathrm{Zu}$ sammenwirken von Psyche und Soma 


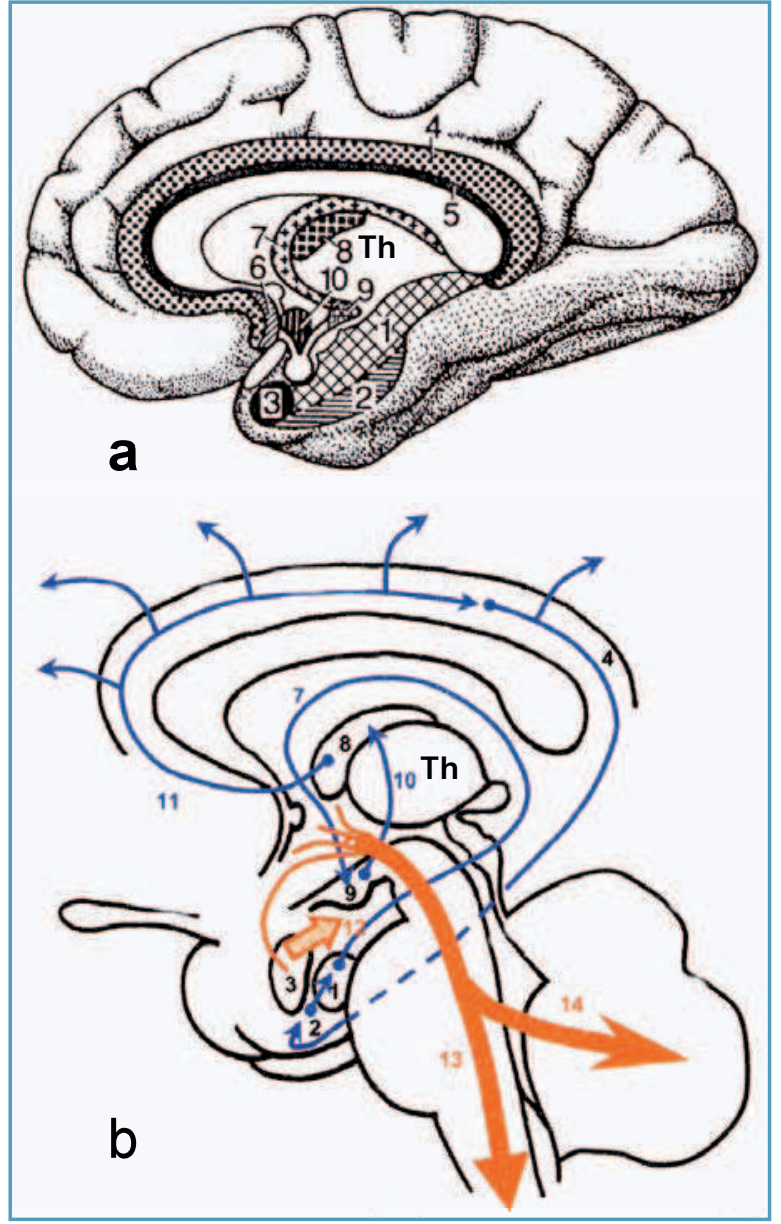

Abb. 1. Lage einzelner Regionen des Limbischen Systems im Gehirn (a). Blaue Bahnen und Pfeile stellen die Faserbahnen des limbischen Systems dar (b). Die roten Bahnen markieren die Verbindungen des Mandelkerns (3) zum limbischen System, Gehirnstamm (13) und Kleinhirn (14); Th Thalamus.

1 Hippocampus, 2 Area entorhinalis, 3 Corpus amygdaloideum (Mandelkern), 4 Gyrus cinguli, 5 Indusium griseum, 6 Area septalis, 7 Fornix, 8 Nucleus anteroir thalami, 9 Corpus mammilare, 10 Tractus mammilothamicus, 11 Cingulum, 12 mediales Vorderhirnbündel, 13 Fasciculus Iongitudinalis dorsalis und 14 Fasern an das Kleinhirn [nach 33].

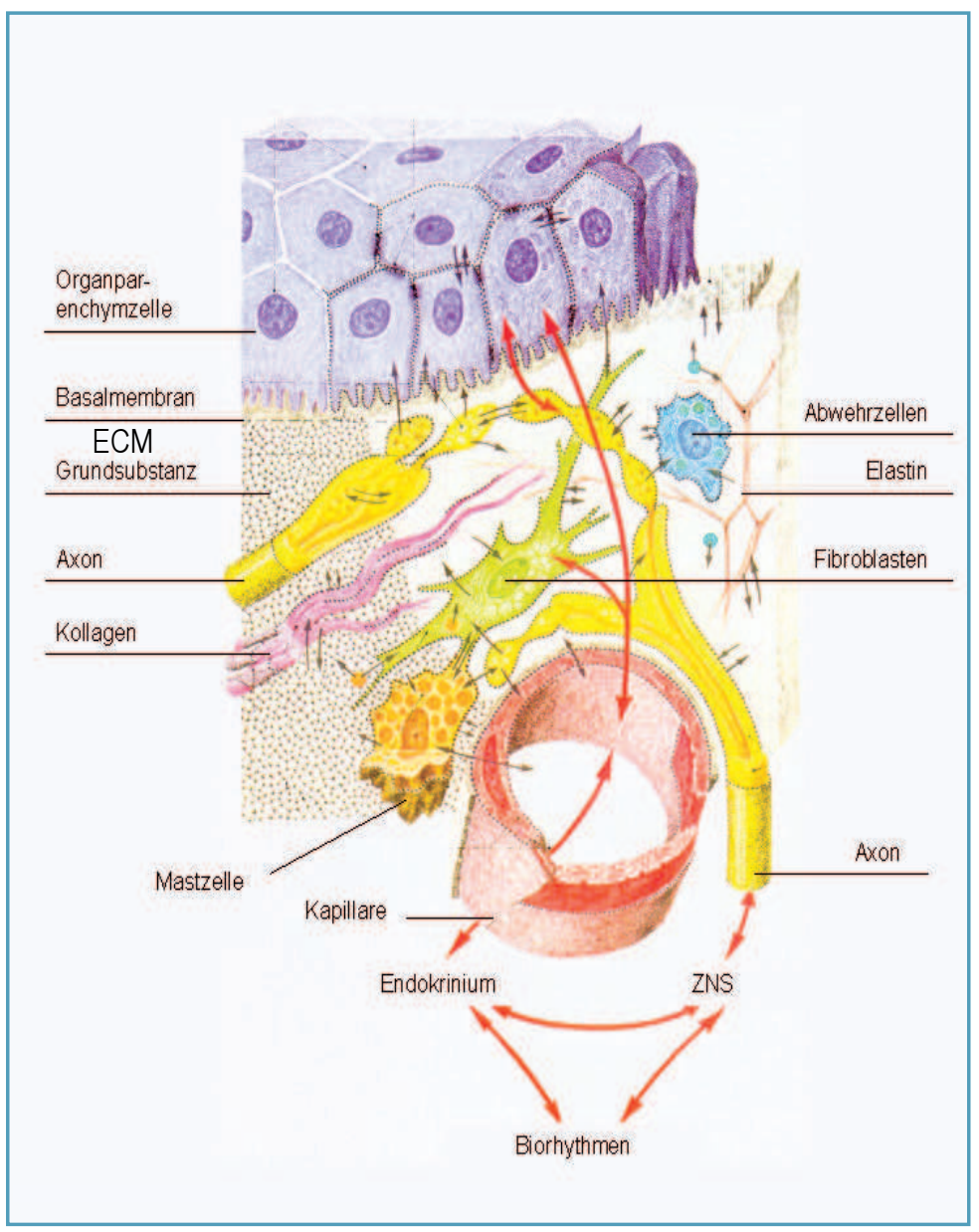

Abb. 2. Schema der Grundregulation. Wechselseitige Beziehungen (Pfeile) zwischen Endstrombahn (Kapillaren, Lymphgefässe), ECM (Grundsubstanz), terminalen vegetativen Axonen, Bindegewebszellen (Mastzellen, AbwehrzelIen, Fibroblasten usw.) und Organparenchymzellen. Epitheliale und endotheliale Zellverbände sind von einer zur ECM vermittelnden Basalmembran unterlagert. Jede Zelloberfläche trägt einen Zuckeroberflächenfilm (Glykokalyx; gepunktete Linien), der zur ECM vermittelt. Die ECM ist über die Endstrombahn an das Endokrinium, über die Axone an das ZNS angeschlossen. Der Fibroblast ist das stoffwechselaktive Zentrum in der Peripherie [nach 14]. bis hin zur Psychoneuroimmunologie erbracht hat, so blieb bisher der Bereich des wechselseitigen Überganges zwischen Psyche und Soma in der konservativen Medizin unbekannt [14, 17].

PISCHINGER [25] hatte bereits in den 50iger Jahren des vergangenen Jahrhunderts nachdrücklich auf die einfache Tatsache verwiesen, dass der Zellbegriff genaugenommen nur eine morphologische Abstraktion wäre. Biologisch gesehen könne er nicht ohne das Lebensmilieu der Zelle genommen werden. Dieses allen Zellen vorgeschaltete Milieu wird durch die Grundsubstanz (extrazelluläre Matrix ECM), bestehend aus Proteoglykanen (PGs), Glykosaminoglykanen (GAGs),
Struktur- und Vernetzungsglykoproteinen (Kollagen, Elastin, Fibronektin u.a.m.), gebildet [14]. Die ECM strukturiert ein Molekularsieb, das über terminale vegetative Nervenfasern an das Zentralnervensystem und über die Endstrombahn an das Endokrinium angeschlossen ist. Beide Systeme sind im Gehirnstamm vielfältig miteinander verschaltet. Dadurch entsteht ein für die Stressverarbeitung hoch effektives „System der Grundregulation“ (Abb. 2) $[14,25]$. Da in der ECM das gesamte Spektrum an Abwehrzellen patrouilliert, ist die Grundregulation auch eng mit dem Immunsystem verknüpft (das Kunstwort Neuropsychoimmunologie gibt dies nicht ausreichend wieder) [17].
Die ECM hält ausserdem die für alle physiologischen Zellfunktionen notwendige elektromagnetische und mechanische Gewebespannung aufrecht und ist raum-zeitlich ein Speicher sowie „Reifungsort“ für informative Zellprodukte u. a. Zytokine und Vorstufen proteolytischer Enzyme [14]. Durch die Fähigkeit der PG/GAGs zur Wasserbindung und Ionenaustausch wird normalerweise in der ECM Isoionie, Isotonie und Isoosmie gewährleistet, eine wichtige Voraussetzung für die Homöodynamik in den Geweben [10, 14].

Auf alle somato-psychischen und psycho-somatischen Eingänge werden die ECM und ihre Komponenten, ohne zwischen „Gut und Böse“ zu unter- 


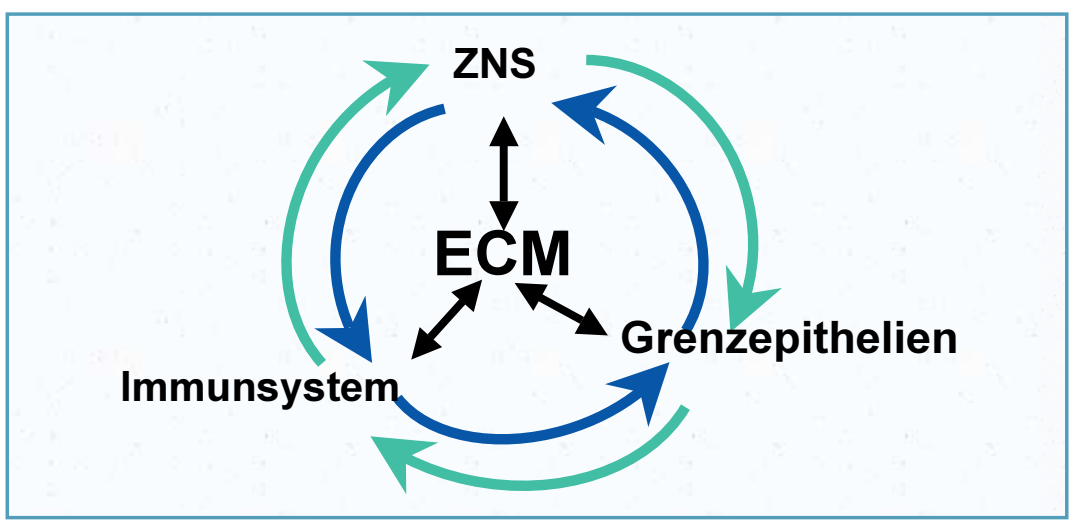

Abb. 3. Bio-psycho-sozialer Funktionskreis.

scheiden, sehr schnell durch qualitative und quantitative Änderungen auf die jeweils aktuelle Situation eingestellt. Dies geschieht über Zellrezeptoren, die einerseits mit der ECM andererseits mit dem Zytofilament- und dem intrazellulären Signaltransduktionssystem (u. a. den MAPKinasen (mitogenaktivierte Proteinkinasen)) in Verbindung stehen [14].

Die die ECM produzierenden Zellen (u. a. Fibroblasten und Astrozyten) reagieren sehr empfindlich auf „Milieuänderungen“ wodurch es über die Grundregulation zu schnellen Adaptationsreaktionen kommt. Das bedeutet: Psyche und Soma laufen in der Grundregulation zusammen. Es gibt keine somatische Krankheit ohne psychische Beteiligung und umgekehrt. Der Körper strebt stets eine biopsycho-sozial zu definierende Homöodynamik an [17, 28].

\section{Stressverarbeitung (Stress- reaktionsprozess SRP)}

Bereits anhaltende Befindensstörungen und die daraus hervorgehenden funktionell-somatischen Störungen sind ein Stressereignis mit erhöhtem Katabolismus der ECM-Komponenten, erhöhtem Anfluten von ROS und Ansäuerung der ECM (latente Gewebsazidose). Das ZNS reagiert auf Stresssituationen mit einem Bewältigungsprogramm, das für die Stabilität und Integrität eines Individuums von entscheidender Bedeutung ist: Bei Konfrontation mit einem Stressor erfolgt zunächst eine subjektive Erstbewer- tung, in der das Ereignis in der Auswirkung auf die eigene Person hin eingeschätzt wird. Die anschliessende Zweitbewertung prüft die eigenen Bewertungsmöglichkeiten und führt $\mathrm{zu}$ einer Neubewertung der Situation. Darauf folgt der Bewältigungsprozess („Coping“) [19]. Dabei ist der SRP durch Rückkopplungen zwischen stresshafter Belastung und ihrer emotionalen sowie mehrphasigen kognitiven Bewertung gekennzeichnet. Diese Situation überwiegt bei Dauerstress (u. a. Beruf, Beziehungsprobleme, Alter, Krankheit). Gelingt es einem Individuum, den SRP aus eigener Kraft zu beenden, liegt ein kontrollierbarer SRP (kSRP) vor, andernfalls ein unkontrollierbarer (uSRP) [19]. Dies hängt ganz vom individuellen bio-psycho-sozialen Funktionskreis ab, der wiederum von der Funktionsfähigkeit der ECM abhängt (Abb. 3). Die ECM ist das „Interface“ der drei Mitglieder des Kreises: Zentralnervensystem, Grenzepithelien (Haut, Cornea, Schleimhäute) und das jeweils zugeschaltete unspezifische und spezifische Immunsystem.

Dabei spielen die vor 10 Jahren entdeckten „Spiegelneurone“ u. a. im Frontalhirn (Brodmann Area 44 und 45), dem vorderen Teil des Gyrus cinguli und der Inselrinde eine wichtige Rolle. Spiegelneurone erstellen den „Spielplan“ einer Handlung. Sie werden aber auch aktiv, wenn man lediglich einen in Grundzügen bekannten Vorgang bei anderen beobachtet (,Einfühlungsvermögen“) [2]. Für den kSRP ist eine seit frühester Kindheit gut eingeübte Spiegelneuronenaktivität äusserst hilfreich, Defizite verstärken dagegen den uSRP.
Während es beim kSRP durch Adaptation an das Geschehen zur Selbstverfügbarkeit der Situation kommt, kann beim uSRP das betroffene Individuum den Vorgang nicht aus eigener Kraft bewältigen. Dabei werden charakteristische neuroplastische Aktivitäten entwickelt [19]: Junge Versuchstiere zeigten bei kSRP eine Vermehrung der Dendritenbäume neokortikaler Pyramidenzellen sowie eine Zunahme von Gliazellen [19]. Im Erwachsenenalter zeichnen sich diese Tiere durch geringe Ängstlichkeit in fremder Umgebung und verminderte Glukokortikoidsekretion aus [19]. Auf den Menschen übertragen bedeutet dies eine individuell rasche Adaptation an stressbedingte Lebensbedingungen. Bei uSRP zeigen die tierexperimentellen neuroanatomischen Befunde, dass aufgrund des anhaltend hohen Glukokortikoidspiegels degenerative neuronale Verschaltungen auftreten [19]. Ein derartiger Destabilisierungsprozess schliesst die Gefahr der Dekompensation des betroffenen Individuums ein, wie dies durch stressinduzierte Erkrankungen (u. a. Psychosen, Herzinfarkt, koronare Herzkrankheit, Magen-Darm Geschwüre) hinlänglich bekannt ist. Das Verlöschen unbrauchbarer Verhaltensmuster bei uSRP hat aber auch den Vorteil, dass sich dann an deren Stelle neue und effektivere Neuronenmuster entwickeln können. Diese Flexibilität im Umgang mit neuen Herausforderungen ist für die Sozialisierung und für Umbruchphasen im Lauf des Lebens wichtig. Ein Mensch, dem es im Laufe seines Lebens gelingt, kSRP und uSRP in ein flexibles Verhaltensmuster $\mathrm{zu}$ bringen, wird belastende Lebenssituationen leichter bewältigen können [19].

Die Stressbewältigung wird jedoch nicht vom ZNS alleine gesteuert, sondern ist in den bio-psycho-sozialen Funktionskreis eingeschlossen (Abb. 3). Besonders die Grenzepithelien werden über ein dichtes Netz somatischer und viszeraler Axone innerviert, in das als Schaltstationen die peripheren Ganglien eingeschaltet sind. Zusätzlich finden sich in den Epithelien und der ECM zahlreiche sogenannte paraneuronale Zellen wie die enterochromaffinen Zellen („helle Zellen System“ Feyerter, „System der diffusen neuro- 
endokrinen Zellen“ [19]. Paraneurone bilden das gleiche Spektrum an Neurotransmittern und neuroendokrinen Peptiden wie sie auch im ZNS gefunden werden. Die freigesetzten Substanzen beeinflussen parakrin die benachbarten Zellen und greifen in alle Belange der Grundregulation ein [9, 14].

Eine besondere Stellung nehmen dabei die Mastzellen ein [21]. Sie werden ebenfalls $\mathrm{zu}$ den Paraneuronen gerechnet [9]. Mastzellen finden sich besonders häufig im Propriabindegewebe von Schleimhäuten. Sie greifen mit ihrem breiten Spektrum an Mediatoren (u. a. neuroendokrine Signalsubstanzen wie Somatostatin, Serotonin, Dopamin, Histamin und vasointestinales Peptid (VIP)) in alle Regelmechanismen des Körpers ein. Mastzellen sind Zwischenglieder übergeordneter nervaler-, endokriner- und immunologischer Regelkreise. Sie dienen der aktuellen raumzeitlichen Feinsteuerung biologischer Aktivitäten und werden daher auch als „Wächter der Grundsubstanz“ bezeichnet [8]. Im Stressverarbeitungsprozess haben Mastzellen aufgrund ihrer Neurotropie zu terminalen Sympathikusaxonen die wichtige Aufgabe, überschiessende Sympathikusreaktionen durch Mediatorenfreisetzung in einen unspezifischen Entzündungsprozess einzubinden, wodurch dann vielfältige Regulationsprozesse in Gang gesetzt werden bzw. therapeutisch zugänglich werden [3, 14].

\section{Periphere Schmerz- verarbeitung bei Stress}

Paraneurone, unspezifische und spezifische Abwehrzellen tragen Azetylcholin(ACh)rezeptoren und stehen in den Geweben in engem zellulären Kontakt zueinander. Sie bilden ein nicht-neuronales cholinerges System (NNAChS), das sich bei allen Mehrzellern findet [30,31]. Auffällig dabei ist, dass Makrophagen/Monozyten als zentrale „Verteiler“ im Immunsystem über nikotinischen ACh-Rezeptoren, die Lymphozyten dagegen über muskarinische verfügen $[15,18]$. Auch die terminalen Sympathikusaxone tragen muskarinische ACh-Rezeptoren [7]; d. h. die Sympathikusfasern werden von den parasympathischen kontrolliert [15]. Bei Säugetieren ist das NNAChS besonders in den Grenzepithelien, Lymph- und Fettgewebe entwickelt. ACh-positiv reagieren neben den genannten Zelltypen auch Astrozyten, Mikrogliazellen, Keratinozyten, Muskelzellen und -fasern sowie Endothelzellen [30, 31]. Die weite Verbreitung des NNAChS wird von einem ubiquitären Auftreten von Azetylcholinesterase begleitet, wodurch ACh stets nur lokal wirksam werden kann. ACh aus nichtneuronalen Zellen ist daher ein „universeller Zytotransmitter“, aber kein Hormon [30, 31]. Freigesetztes ACh wirkt autokrin auf die freisetzende Zelle zurück und steuert so seinen eigenen Bedarf; parakrin wirkt es auf die umgebenden Zellen und terminalen vegetativen Axone. Dabei bilden die Zellen des NNChS untereinander dynamische Zellkontakte und mit terminalen vegetativen Axonen „Neuroeffektorbezirke“ sogenannte Synapsen auf Distanz [8, 23]. Dadurch können Informationen sehr schnell weitergeleitet und bereits vor „Ort“ sortiert werden. In diesem Zusammenhang wurde von HeINE [15] darauf hingewiesen, dass das Mesenchym, das das Nerven-Gefässbündel eines Akupunkturpunktes begleitet, besonders reich an derartigen NNACh-Beziehungen ist.

Noradrenalin (NA) aus den terminalen Sympathikusfasern initiiert Mastzelldegranulation, wobei u. a. auch die stark proinflammatorischen Zytokine Tumornekrosefaktor-alpha (TNF- $\alpha$ ) und Interleukin-12 (IL-12) freigesetzt werden. In gleicher Weise reagieren Makrophagen/Monozyten obwohl diese Zellen gleichzeitig dem NNAChS angehören. Das über das NNAChS gebildete wie auch aus terminalen parasympathischen Axonen freigesetztes ACh zügelt wiederum die stressbedingte Zunahme an NA. Dazu tragen auch die aus Th2 (Th Helfer T-Lymphozyten) und regulatorischen T-Zellen (Th3 bzw. Treg Zellen) freigesetzten entzündungshemmenden Zytokine TGF- $\beta$, (Transforming Growth Factor-beta), IL-4 und IL-10 bei [Literatur-Übersichten in 14,18$]$.

Alle Mediatoren reagieren über die ECM mit entsprechender Reaktion des
Systems der Grundregulation [14, 25]. Stressmediatoren wie Katecholamine und proteolytische Enzyme führen aufgrund der Bildung saurer ECM Spaltprodukte und von ROS zu einer latenten Gewebsazidose mit Selbstverstärkung des Stressprozesses [LiteraturÜbersichten in14, 16].

Eine Bremse in diesem Geschehen bilden im ZNS wie auch in der Peripherie ubiquitär gebildete Endocannabinoide (amidierte Lipide). Sie werden von Makrophagen und Lymphozyten (im ZNS von Gliazellen und Microgliazellen) gebildet [5]. Ihre Synthese wird durch NA erhöht. Die in der Peripherie gebildeten Endocannabinoide haben einen eigenen, vom zentralnervösen Endocannabinoid-Rezeptor (CB1) verschiedenen Rezeptor (CB2), der sich u. a. auch auf nozizeptiven Axonen befindet [13, 23]. Ausserdem können Endocannabinoide heterozeptiv die auf Schmerzfasern gelegenen exzitatorischen Vanilloidrezeptoren (Abbauprodukte von NA und Adrenalin) besetzen [24]. Endocannabinoide wirken daher schmerzlindernd und antiinflammatorisch, wodurch sie zur Minderung der Stresslast beitragen $[13,22]$. Von besonderer Bedeutung sind dabei das Anandamid (AEA; Ethanolamid der Arachidonsäure) und 2-Arachidonylglycerol (2-AG). Mit AEA wird gleichzeitig das verwandte Molekül Palmitylethanolamid (PEA) freigesetzt [5, 20, 22, 26]. Diese Substanzen werden durch die Enzyme Fettsäureamidgydrolase (FAAH) und Monoacylglycerollipase (MAGL) schnell abgebaut [4, 6, 22]. Die Bedeutung von PEA liegt in seiner Fähigkeit die Mastzelldegranulation $\mathrm{zu}$ hemmen [5].

Wie das NNAChS stellen daher auch die Endocannabinoide ein ubiquitäres Zytotransmittersystem dar. Das Endocannabinoid- und NNACh-System sind daher entscheidende Faktoren der Grundregulation, vor allem bei schmerz- und damit stressbelasteten Vorgängen. Auch hier ist wieder die ECM der entscheidende funktionelle Faktor. Gerät die ECM durch anhaltende unphysiologische Belastungen in eine latente Azidose, werden diese beiden ubiquitären „Stresszügel“ überfordert mit drohender Entwicklung chronischer Krankheiten [14, 16]. 


\section{Neuraltherapie und Akupunktur in der Stressverarbeitung}

Beide Therapieverfahren führen im Stressgeschehen zu einer Gegenirritation. Diese wird umso nachhaltiger ausfallen, je besser eine meist vorhandene latente Gewebsazidose zurückgedrängt werden kann. Diese ist qualitativ leicht aus dem Harn zu messen (Einzelheiten [16, 32]).

Bei Neuraltherapie wird der Sympathikus direkt durch Lokalanästhetika gebremst. Wie von MACCARONE et al. [20] am Beispiel von Procain gezeigt wurde, kommt dessen analgetisch-anästhetischer Effekt u. a. durch lokale Erhöhung des Endocannabinoidspiegels zustande. Lokalanästhetika wie Procain werden in der ECM durch Hydrolasen in Aminoethylethylate und Benzylamide gespalten. Aus Procain entsteht dabei Diethylaminoethylat (DEAE) und p-Aminobenzoesäure. DEAE bindet an die bei entzündlichen Prozessen aus Zellmembranen durch Phospholipase A generierten Arachidonsäurederivate. Dadurch entstehen Endocannabinoidanaloga, die durch FAAH und MAGL zwar gebunden aber durch sterische Hemmung nicht abgebaut werden können. Dies wurde von HEINE [13] entsprechend der Untersuchungen von BRACEY et al. [4] zur räumlichen Struktur von FAAH gefolgert. Zwangsläufig steigen dann lokal im Bereich der Procaingabe die Endocannabinoide an und können dort auch die Vanilloidrezeptoren der Schmerzfasern besetzen. Die lokale Beruhigung wird dann über den segmentalregulatorischen Komplex in die gesamte Grundregulation eingespeist $[13,14]$, wodurch der übersteigerte Sympathikotonus reguliert wird. Im Unterschied dazu aktiviert Akupunktur u. a. den Parasympathikus und damit auch das NNAChS. Dadurch wird eine übersteigerte Sympathikuswirkung zurückgedrängt [15, 18].

Akupunktur wie auch Neuraltherapie sind daher jeweils auf ganz eigene Weise in der Lage, bereits in der Peripherie eine Schmerzverarbeitung einzuleiten, die dann, nach Zentral geleitet, auf den bio-psycho-sozialen Funktionskreis im Sinne eines Stressabbaues zurückwirkt.

\section{Schlussfolgerung}

Belastungsstörungen als Stressphänomene sind mit Irritationen im bio-psycho-sozialen Funktionskreis verbunden. Dieser Kreis ist in das System der Grundregulation eingebettet. Psyche und Soma scheinen daher in der Regelung der Grundsubstanz (ECM) zusammenzulaufen. Entsprechend sind im Stressbewältigungsprozess Neuroplastizität und Reaktivität der ECM immunologisch, nervös und endokrin miteinander verbunden. Da im Stressgeschehen der Sympathikus überaktiviert ist, gerät die Grundregulation in einen proinflammatorischen Zustand. Dadurch wird die ECM latent azidotisch mit Selbstverstärkungseffekten auf das Stressgeschehen. Zur Entsäuerung der ECM sind Ernährungsumstellung und psychische Entspannungsübungen geeignet. Unter diesen Bedingungen kann durch Neuraltherapie der Symphatikus zurückgedrängt, durch Akupunktur der Parasympathikus gestärkt werden. Diese periphere Vorarbeit ist notwendige Voraussetzung für die geregelte Schmerzverarbeitung im ZNS.

\section{Fazit}

Neuraltherapie und Akupunktur eignen sich zur Stresstherapie. Während die Akupunktur u. a. über eine Stärkung des Parasympathikus wirkt, hemmt die Neuraltherapie insbesondere überschiessende Sympathikuswirkung. Allerdings muss zuvor auf latente Gewebsazidose geprüft und diese gegebenenfalls vor- bzw. mittherapiert werden, da sonst regulationsmedizinische Verfahren nicht greifen können.

\section{Literatur}

1. Aaron LA, Buchwald DA. A review of the evidence for overlap among unexplained clinical conditions. Ann. Intern. Med. 2001:134:868-881.

2. Bauer J. Warum ich fühle, was du fühlst. Hamburg: Hoffmann und Campe. 2005.

3. Berg PA. Neuroimmunologischze Aspekte funktioneller somatischer Syndrome. Dtsch. Med. Wochenschr. 2005;130:107-113.

4. Bracey M, Hanson M, Masuda KR et al. Structural adaptations in a membrane enzyme that terminates endocannabinoid signaling. Science. 2002:298:1703-1796.

5. Calignano A, La Rana G, Giuffrida A, Piomelli D. Control of endogenous cannabinoids. Nature. 1998;394:277-281.

6. Di Marzo V, Bisogno $T$, De Petrocellis $L$ et al. Biosynthesis and inactivation of the endocannabinoid 2- arachidonylglycerol in circulation and tumoral macrophages. Eur. J. Biochem. 1999;264:258-267.
7. Elenkov IJ, Wilder RL, Chrousos GP, Vizi ES. The sympathetic nerve - an integrative interface between two supersystems: the brain and the between two supersystems: the brain and the
immune system. Pharmacol. Rev. 2000;52:595-638.

8. Förster FJ, Heine H, Schaeg G. Histophysiology of the vegetative peripheral nervous system of skin. Arch. Derm. Res. 1975;254:295-302.

9. Fujita T, Kanno T, Kobayashi S. The Paraneuron. Tokyo, Berlin, Heidelberg: Springer-Verlag, 1988.

10. Hauss WH. Die unspezifische Mesenchymreaktion (UMR). Das essentielle Ereignis der in den Industriestaaten häufigsten Erkrankungen. Perfusion 1994;9:312-322.

11. Heine $\mathrm{H}$ : Psychoneuroimmunologie und Grundregulation - eine Übersicht: In: Stacher A Hrsg Ganzheitsmedizin und Psychoneuroimmunologie. Vierter Wiener Dialog. Wien: Facultas 1997:50-56.

12. Heine H. Gesundes Altern: Bedeutung der Grundregulation. Geriatrie Journal. 2002; Heft 10:44-46 und Heft 11:37-40.

13. Heine $\mathrm{H}$ : Wirkt Neuraltherapie nach Huneke übe das periphere Endocannabinoidsystem? Ärztedas periphere Endocannabinoidsystem? A

14. Heine H. Grundregulation - Eine Synthese medizinischen Denkens. Dtsch. Ztschr. f. Akup. 2004;47:6-19.

15. Heine H. Periphere Schmerzverarbeitung an $\mathrm{Ge}$ lenken durch Akupunktur - Bedeutung des Parasympathikus. Dtsch. Zschr. Akup. 2004:47:15-23.

16. Heine $H$. Die extrazelluläre Matrix als Attraktor für Verschlackungsphänomene. Ärztezeitschr. f Naturheilverf. 2005;46:256-260.

17. Heine $H$. Stressverarbeitung: Bedeutung in der Medizin. Zu den Beiträgen aus DMW 3/2005. Dtsch. Med. Wochenschr. 2005:130: 905-906.

18. Heine H. Die Bedeutung der Grundregulation für Neuraltherapie, Akupunktur und Homöopathie. Erfahrungsheilkunde 2005;54:170-175.

19. Huether G. The central adaptation syndrome: Psychosocial stress as a trigger for adaptive modifications of brain structure and brain function. Progr, Neurobiol. 1996;48:569-612.

20. Maccarone $M$, De Petrocellis L, Bari $M$ et al. Lipopolysaccharide downregulates fatty acid amid hydrolase expression and increases anandamide levels in human peripheral lymphocytes. Arch. Biochem. Biophys. 2001; 393:321-328.

21. Mach T. The brain-gut axis in irritable bowel syndrom - clinical aspects. Med. Sci. Monit. 2004 10:RA 125-131.

22. Marx J. Drugs inspired by a drug. Science 2006;311;322-325 (NEWS Focus).

23. Merwe P. Davis SJ. The immunological synapse - a multitasking system. Science 2002;295: 1479-1480.

24. Pertwee RG. Cannabionoid receptors and pain Prog. Neurobiol. 2001;63:569-611.

25. Pischinger A. Das System der Grundregulation Grundlagen für eine ganzheitsbiologische Theorie der Medizin. Heine H Hrsg. 10. überarbeitete Aufl. Stuttgart: Haug, 2004

26. Randall MD, Kendall DA. Endocannabinoids: new class of vasoactive substances. Trends. Pharmacol. Sci. 1998:19:55-58.

27. Schipperges H. Medizin an der Jahrtausendwende. Fakten, Trends, Optionen. Frankfurt: Jose Knecht, 1991

28. Schüffel W. Stressverarbeitung: Bedeutung und Sinn in der Medizin. Dtsch. Med. Wochenschr. 2005:130:85-86.

29. Selye H. Einführung in die Lehre vom Adaptationssyndrom. Stuttgart: Thieme, 1952.

30. Wess J, Duttaroy A, Gomeza J et al. Muscarinic receptor subtypes mediating central and peripheral antinociception studied with muscarinic receptor knockaut mice: a review. Life Sci. 2003:72:2047-2054

31. Wessler I, Kilbinger $H$, Bittinger $F$ et al. The biological role of non-neuronal acetylcholine in plants and humans. Jpn. J. Pharmacol. 2001;85:2-10.

32. Worlitschek M. Die Praxis des Säure-Basenhaushaltes. 5. Aufl. Heidelberg: Haug Verlag, 2001.

33. Zilles K, Rehkämper G. Funktionelle Neuroanatomie. 3. Aufl. Berlin, Heidelberg, New York: Springer, 1998.

\section{Korrespondenzadresse:}

Prof. Dr. rer. nat. med. habil. Hartmut Heine Billerbeckweg 1-3, DE-75242 Neuhausen

hartmutheine@aol.com 